\title{
Chemical Pneumonitis Following Household Exposure to Hydrofluoric Acid
}

\author{
James R. Bennion, MD, MPH, Maj USAF, and Alfred Franzblau, MD*
}

\begin{abstract}
A previously healthy 26-year-old woman developed hemorrhagic alveolitis and adult respiratory distress syndrome (ARDS) following use of a hydrofluoric acid-containing household cleaning agent. Exposure was inhalational in nature. The circumstances and possible mechanism of chemical pneumonitis from low-dose inhalational exposure to hydrofluoric acid are discussed. Am. J. Ind. Med. 31:474-478, 1997. ( 1997 Wiley-Liss, Inc.
\end{abstract}

\section{KEY WORDS: hydrogen fluoride; CAS\#7664-39-3; chemical pneumonitis; adult respiratory distress syndrome (ARDS); household products; inhalation injury}

\section{INTRODUCTION}

Hydrogen fluoride (HF) is a weak acid existing as an associated molecule up to $\mathrm{H}_{6} \mathrm{~F}_{6}$ at $1 \mathrm{~atm}$ with temperatures of $<100^{\circ} \mathrm{C}$; under these conditions, the average molecular weight of the molecules is 50-55 Daltons [Stokinger, 1981]. It is infinitely soluble in cold water [Handbook of Physics and Chemistry, 1978] and fumes strongly in moist air [Stokinger, 1981].

$\mathrm{HF}$ is used in a variety of industrial processes involving brick, glass, ceramics, plastics, aluminum, ferroenamels, fluorocarbons, aerosols, welding, coal burning, rocket engine fuels, high octane fuels, silicone wafer etching, dental laboratories, ore extraction processes, phosphate fertilizers, insecticides, and cleaning agents [Caravati, 1988; Dieffenbacher and Thompson, 1962; MacKinnon, 1988; Machle and Kitzmiller, 1934; Machle et al., 1934; Mayer and Gross, 1985; Stokinger, 1981; Waldbott and Lee, 1978]. HF is usually produced by interaction of calcium fluoride (fluorspar) and sulfuric acid [MacKinnon, 1988]. For HF gas, the American Conference of Governmental Industrial Hygienists ceiling limit is $3 \mathrm{ppm}$ as F [ACGIH, 1994]. The Occupational Safety and Health Administration's Permis-

Department of Environmental and Industrial Health, School of Public Health, University of Michigan, Ann Arbor, MI.

Dr. Bennion is now at Occupational Medicine Services, 78th Medical Group/SGPFO, 655 7th Street, Robins Air Force Base, GA 31098-2227.

*Correspondence to: Dr. Franzblau, Department of Environmental \& Industrial Health, School of Public Health University of Michigan, Ann Arbor, MI 48109-2029.

Accepted for publication 16 September 1996. sible Exposure Limit and National Institute for Occupational Safety and Health's Recommended Exposure Level are both $3 \mathrm{ppm}$ as well. The NIOSH Immediately Dangerous to Life and Health Level is $30 \mathrm{ppm}$ [Alexeeff et al., 1993].

Most literature related to HF injury pertains to dermal contact. Inhalation injury due to HF has usually occurred in industrial and laboratory settings, although at least one forensic case has been documented [Chela et al., 1989]. Almost all documented cases resulting in significant pulmonary pathology have occurred following massive exposures [Braun et al., 1984; Caravati, 1988; Greendyke and Hodge, 1964; MacKinnon, 1988; Mayer and Guelich, 1963; Sadove et al., 1990; Trevino et al., 1983; Watson et al., 1973; White and Templeton, 1992]. We describe a case of chemical pneumonitis following use of a household cleaning agent containing HF. To our knowledge, pulmonary injury associated with use of a household product containing HF has not been reported.

\section{CASE REPORT}

A previously healthy 26-year-old woman used multiple household products in an attempt to remove a stain from a bathtub (see Table I for details on product contents). She had a past medical history of "walking pneumonia" diagnosed without confirmatory chest radiograph a year preceding this illness, and a 13-year history of semiannual episodes of acute bronchitis. All these illnesses had resolved with antibiotic therapy. She also smoked 1.5 packs of cigarettes a day for the same 13 years. Her occupational history was unremarkable for significant pulmonary expo- 
TABLE I. Active Ingredients of Household Cleaning Products

\begin{tabular}{|c|c|}
\hline Product & Active Ingredient \\
\hline X-14 Mildew Stain Remover ${ }^{\circledR}$ & 25 to $45 \%$ sodium hypochlorite \\
\hline The Works Tub and Tile Cleaner ${ }^{\circledR}$ & $18 \%$ phosphoric acid \\
\hline Ammonia & 2 to $2.5 \%$ ammonia hydroxide \\
\hline Liquid bleach & $5.25 \%$ sodium hypochlorite \\
\hline Sno-bol ${ }^{\circledR}$ & $14.5 \%$ hydrochloric acid \\
\hline White vinegar & $5 \%$ acetic acid \\
\hline Whink Rust Remover ${ }^{\circledR}$ & $8 \%$ hydrofluoric acid \\
\hline
\end{tabular}

sures: she had not been working for 2 months preceding her illness and had previously been an in-house care giver. She did not use any medications. She was $178 \mathrm{~cm}$ tall and weighed $115 \mathrm{~kg}$.

The tub was located in a small bathroom (approximately $5 \times 7 \times 7$ feet) with no ventilation other than a heating vent. It was late fall, and the only window was sealed shut with plastic. The door to the bathroom was kept closed when cleaning the tub. At no time did she mix different products, and she always rinsed the tub with cold water before applying each different product. She wore no gloves, using an $\mathrm{Ajax}^{\circledast}$ pad initially and thereafter a stiff, long-handled brush.

On day 1, she used Comet ${ }^{\circledast}$ and an Ajax ${ }^{\circledast}$ pad, then $10 \mathrm{oz}$ of X-14 Mildew Stain Remover ${ }^{\oplus}$. On day 2, she tried $32 \mathrm{oz}$ of The Works Tub and Tile Cleaner ${ }^{\circledR}$; this was followed by 8 oz of ammonia. After the usual cold water rinse, she next plugged the drain and poured in a gallon of liquid bleach, then adding cold water. She let this sit for several minutes before allowing to drain. On day 3, she applied $16 \mathrm{oz}$ of Sno-bol ${ }^{\oplus}$. She next applied $16 \mathrm{oz}$ of white vinegar. Later, she applied a half-gallon of liquid bleach. As stated above, each application was followed by a cold water rinse. She and the other household residents did not recall any symptoms of mucous membrane irritation up to this point in time.

At 10 PM on day 3, she applied $10 \mathrm{oz}$ of Whink Rust Remover $^{\circledast}$ over an estimated 30 minute interval. During this application she experienced burning and tearing to both eyes and a foul taste to her mouth; she did not recall experiencing any upper or lower respiratory symptoms at that time. However, two other household members who both used the bathroom shortly after she applied Whink Rust Remover ${ }^{\circledast}$ recalled experiencing strong, almost unbearable eye, nose and throat irritation.

Following the initial application, she went to a store and obtained 2 more 10-oz bottles of Whink Rust Remover ${ }^{\circledR}$, which she applied over an additional estimated 30-min interval that same evening. During the second application of Whink $^{\circledast}$ she experienced a burning sensation to the eyes and a foul taste to her mouth (the latter persisted until breakfast the following morning). She did not experience any dermal burns.
On day 4, the patient was "sleepy" and remained in bed much of the day; she did not recall experiencing respiratory symptoms through day 4 . She awoke earlier than usual the morning of day 5 with right lateral chest pain, shortness of breath, and a nonproductive cough. She had not yet experienced any fever, chills or sweats.

Before 8 AM on day 6 , she was seen in the emergency department of a local hospital. Her vitals signs were remarkable for a respiratory rate of $28 / \mathrm{min}$ and a temperature of $36.2^{\circ} \mathrm{C}$. Auscultation revealed rales and wheezes over the right chest. The white blood cell (WBC) count was 14,000 with a normal differential; arterial blood (ABG) gas values were unremarkable. A chest radiograph showed a right lower lobe infiltrate. Refusing admission, she was prescribed cephalexin monohydrate and ibuprofen and sent home.

She returned to the same hospital and was admitted the evening of day 7 , complaining of severe right shoulder pain, increased nonproductive cough, orthopnea, fevers, and chills. Her vital signs were recorded as temperature $35.3^{\circ} \mathrm{C}$, pulse 100, blood pressure 110/80, and respirations 40/min. Her nose and throat were documented as "clear." Auscultation revealed diffuse rhonchi and wheezing over both lungs. The leukocyte count was 13,000 with $76 \%$ segmentals, $15 \%$ lymphocytes, $7 \%$ monocytes, and $2 \%$ eosinophils. A repeat chest radiograph again demonstrated a right lower lobe infiltrate. Treatment was continued with cephalexin monohydrate and oxygen.

On day 8, electrolytes, blood urea nitrogen (BUN), and creatinine were normal. The leukocyte count was 14,000 with a normal differential. A chest radiograph obtained at 4:08 PM showed loss of the right heart border, with infiltrate involving all lobes except the right upper lobe. At 5 PM she was transferred to the coronary care unit (CCU), where she was diuresed $2 \mathrm{~L}$ with Lasix and was started on erythromycin, clarithromycin, cefuroxime sodium, and methylprednisolone sodium succinate (60 mg IV q6h). Her respiratory rate reached 45/min, and she was intubated later the same evening.

At 1 AM of day 9, she experienced a sudden decrease in oxygenation, requiring $100 \% \mathrm{O}_{2}$, and $10 \mathrm{~cm} \mathrm{H}_{2} \mathrm{O}$ of peak end-expiratory pressure (PEEP) to maintain oxygen saturation above $70 \%$. Later on day 9 , she was transferred to the University of Michigan Medical Center (UMMC). She remained on methylprednisolone sodium succinate $60 \mathrm{mg}$ q6h through the morning of day 10 ; then she received $1 \mathrm{~g}$ of methylprednisolone sodium succinate each day for 3 more days. She was kept on erythromycin and began ceftriaxone and gentamycin. She was maintained on antibiotic therapy for the remaining 28 days of her admission at UMMC.

She experienced fevers of $<38.3^{\circ} \mathrm{C}$ on day 9 and several times during the next 2 weeks. Bronchoscopy with brushings and lavage, accomplished on day 9, was consistent with alveolar hemorrhage. Stains and cultures for 
TABLE II. Spirometry Results Following Hospitalization for ARDS

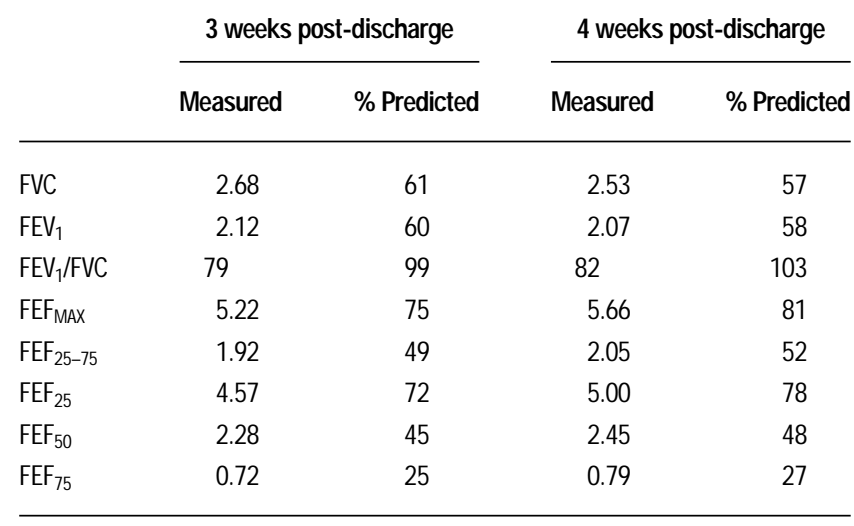

bacteria, fungi, mycobacteria, and viruses were all negative. Physical examination on day 10 revealed no rash, discoloration, or blistering to the upper extremities. Connective tissue disease tests were unremarkable (i.e., negative antinuclear antibody, antinucleolar autoantibody, and anti-doublestranded deoxyribonucleic acid assays). A chest computed tomogram (CT) performed on day 19 showed focal bilateral lower lobe airspace disease with extensive ground-glass appearance of the upper lung zones-left lung greater than right. A second bronchoscopy was accomplished day 21; the results were significant for "yeast, not Cryptococcus," and the patient was treated with IV fluconazole. A polymerase chain reaction (PCR) test of bronchial washings for tuberculin deoxyribonucleic acid was negative. Cultures for varicella, cytomegalovirus and respiratory viruses tests were all negative.

She was extubated on day 25 but remained hospitalized for treatment of a deep venous thrombosis and heme positive stools through day 36 . One month after discharge, she had a persistent, nonproductive nocturnal cough and dyspnea on climbing one flight of stairs; she had not resumed smoking. Pulmonary function test results were consistent with mixed mild restrictive disease and with mild to moderate obstruction in small airways (Table II).

No measurements of serum calcium were performed at the outside hospital. Serum calcium was measured at UMMC on day 9, 6 days after exposure to the Whink ${ }^{\circledR}$, and was normal.

\section{DISCUSSION}

This patient experienced hemorrhagic alveolitis and adult respiratory disease syndrome (ARDS). The etiological factor from her history that best explains the etiology of her illness is her exposure to HF. There is reason to believe her inhalational exposure to $\mathrm{HF}$ was adequate to initiate the injury, and there is evidence to support a proposed mechanism for such an injury.

To summarize the case history, the patient experienced immediate irritant symptoms following use of an $\mathrm{HF}$ - containing product to clean her bathtub. Within 2.5 days, she was documented to have cough, chest pain, and a right lower lobe infiltrate on chest radiography. After 2.5 more days, while on oral and then intravenous antibiotic therapy, her condition worsened with the development of ARDS, requiring prolonged mechanical ventilatory support. Early bronchoscopy (day 9) showed the presence of hemmorrhagic alveolitis. Upon recovery she was left with a residual mixed restrictive/obstructive pulmonary deficit.

Although it is possible that the patient's pathology was caused by a biological or chemical agent other than HF, HF remains the most likely causative factor. The patient had no recent history of exposure to illness. Testing and cultures for a biological cause were negative, and she did not respond to antiobiotic therapy. Although the early chest radiographs showed a focal lesion suggestive of an infectious agent, such focal lesions have been found to occur in animal subjects exposed to HF [Machle and Kitzmiller, 1934; Stokinger, 1949]. The exposure in this case was of an intensity closer to that of those animal subjects than to the massive exposures described in most recorded human inhalation injuries. Additionally, focal pneumonias are a known complication of $\mathrm{HF}$ inhalational injury in animal subjects [Machle and Kitzmiller, 1934]. Also, given the lower intensity of $\mathrm{HF}$ exposure experienced by the patient, it is possible that her ARDS evolved more slowly than has been recorded among cases with massive, overwhelming exposure.

It is doubtful that the non-HF cleaning agents contributed to the patient's pulmonary injury. The cold water rinse between her use of ammonia hydroxide and her use of sodium hypochlorite on day 2 would have prevented the formation of chloramines [Reisz and Gammon, 1986]. The patient specifically denied ever mixing any of the different cleaning agents. Further, neither the patient nor the other household occupants recalled experiencing irritation on exposure to any of the non-HF containing products. And, the chemical agents in the non-HF products would not be expected to cause delayed-onset chemical pneumonitis as is known to occur following exposure to HF [Braun et al., 1984].

Although HF exposure levels experienced by the patient

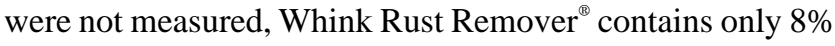
HF. It has been suggested that inhalation hazard may not exist in solutions containing less than 60\% HF [Mayer and Guelich, 1963]. Other publications suggest inhalation injury due to HF in the absence of massive sudden exposure or entrapment is unlikely. It is thought that the noxious effects of gaseous $\mathrm{HF}$ at concentrations adequate to induce pulmonary insult would drive exposed individuals away before significant injury could occur [White and Templeton, 1992]. These opinions appear to have been derived from experience with brief, immediately life-threatening industrial exposures rather than more prolonged and moderate exposures. The exposure experienced by our patient had several unique features that suggest a mechanism whereby she could have 
experienced an unsafe exposure to HF, which produced a more delayed, rather than an immediate, clinical response.

The patient was working in a small, unventilated room, and she used 3 full bottles of Whink ${ }^{\circledast}$ in a short interval. Further, she was physically active, scrubbing the HFcontaining product in a bathtub. The scrubbing action would have promoted aerosolization of the HF solution; it would also have generated some heat, increasing the volatilization of the HF. As HF and mist are heavier than air, and as no significant ventilation was present, these would have been at a greater concentration within the bathtub itself. The patient was working with her head directly over or even partially within the bath tub, so she would have inhaled this higher concentration of HF and HF-containing aerosol. Additionally, her physical activity would have increased the rate and depth of her respirations and would have predisposed her to mouth breathe.

Many human nose breathers will mouth breath during physical activity [Stavert et al., 1991]. Exercise and oronasal and oral breathing have been associated with increased penetration of highly water soluble chemicals to the deep lung [Dungworth, 1989; Miller et al., 1989]. In bypassing the nasal passageways, a significant protective mechanism of the lungs is eliminated.

HF gas would normally (i.e., in the absence of physical activity) be expected to deposit in the human nasal passageways preferentially for several reasons. Gaseous HF is infinitely soluble in cold water [Handbook of Chemistry and Physics, 1978], and it has been observed that gases with higher solubility tend to be scavenged effectively by the upper respiratory mucosa, causing upper respiratory injury. Intermediately soluble, and highly insoluble toxic gases tend to have their effect on the lower respiratory mucosa [Reisz and Gammon, 1986; White and Templeton, 1992; Morris and Smith, 1982; Stavert et al., 1991; Dungworth, 1989; Miller et al., 1989]. Additionally, more reactive compounds are thought to be more effectively scavenged in the upper airways, and $\mathrm{HF}$ is very reactive at physiological $\mathrm{pH}$, being a weak acid (pK 3.45).

Once in contact with lung tissue, HF would most probably cause cell injury via the same two mechanisms thought to cause damage in dermal exposures: first, by acting as a weak acid; and second, by formation of fluoride salts with intracellular calcium and magnesium-leading to cell death [MacKinnon, 1988].

The delay between exposure and pulmonary symptoms in the case we describe is consistent with reported HF inhalation injuries. With massive HF exposures, pulmonary injury was evident in a man who died within 30 minutes of exposure [Watson et al., 1973]. However, intraluminal exudate can be most severe 2-3 days after exposure to a reactive gas [Dungworth, 1989; Miller et al., 1989]. And, death secondary to inhalation of HF has been reported to occur days to weeks after injury [Braun et al., 1984]. We hypothesize that with massive exposure the primary mechanism of tissue injury is related to the acid properties of HF. At lower levels of exposure, and if the patient survives the effects of the acidic insult, salt formation by fluoride ions may become more important in determining local pulmonary tissue damage. In the present case exposure was not overwhelming, but we believe it was adequate for the fluoride ion to produce serious pulmonary injury.

That the patient was exposed to HF capable of causing her pulmonary injury is further supported by the symptoms experienced by herself and the other two adult occupants of the house. She experienced a foul taste and eye irritation but does not recall experiencing the nose and throat irritation experienced by the other two adults, who visited the bathroom near the time of the Wink ${ }^{\circledast}$ product application. All four of these symptoms have been documented to occur at $\mathrm{HF}$ concentrations as low as $12.1 \mathrm{ppm}$ HF [Machle et al., 1934]. A foul taste, described as flat and sour by Machle, has been reported to occur at levels at least as low as $5.9 \mathrm{ppm}$ [Largent, 1961].

That the patient did not recall having experienced the irritation described by the other two adults might be accounted for by the known difference in symptoms experienced by different subjects at specific concentrations of HF [Largent, 1961]. However, the other two adults had exposures of only a few minutes, a fraction of the time experienced by the patient; with her more prolonged exposure, she may have become acclimatized to the HF. Such an acclimatization has been well documented in human studies [Collings et al., 1951]. Acclimatization to HF has been further confirmed in a study where animals exposed to 23.3 ppm HF were observed to experience mild respiratory tract irritation which decreased after 5-15 min, when the animals developed a mucoid discharge [Machle et al., 1934].

Whether or not acclimatization occurred, a concentration of $7.1 \mathrm{ppm}$ HF has been determined to be "tolerable and respirable" in one human study involving brief exposures, and higher concentrations were felt to be sufficiently irritating as to discourage prolonged human exposure [Machle and Kitzmiller, 1934]. The question of whether an exposure to $7.1 \mathrm{ppm}$ HF could cause pulmonary injury in an exposure similar to that experienced by the patient in this paper is not answered by the human studies cited in this paper. However, animal studies suggest a potential for injury.

Stokinger [1949] found moderate hemorrhage and edema in the lungs of 3 of 4 dogs exposed to $11.6 \mathrm{ppm}$ HF gas for 6 $\mathrm{hr} /$ day over a 5 -week period and that 1 of 5 dogs exposed to $3.3 \mathrm{ppm}$ had a focal area of pulmonary hemorrhage. At lethal levels, death resulted from pulmonary damage, and at nonlethal levels, if any damage occurred it was most frequently pulmonary damage.

Machle and Kitzmiller [1934] exposed 1 Rhesus monkey, 5 rabbits, and 3 guinea pigs to 7.08 ppm HF for 6 or 7 $\mathrm{hr} / \mathrm{day}$ for 50 days (excluding weekends) for a total 309-hr 
exposure. The monkey suffered renal damage, but had no pulmonary lesions. One guinea pig died after a 134-hr exposure and was found to have a "low-grade inflammatory reaction in the alveolar walls with atelectasis, and pronounced degenerative changes and hyperplasia of the bronchial epithelium." A second guinea pig died at $160 \mathrm{hr}$ and was found to have "a large, partly organized pulmonary hemorrhage with low-grade cellular reaction." The third guinea pig was sacrificed 9 months after exposure and was found to have organized pulmonary hemorrhages, alveolar exudates, and cellular infiltrations of the alveolar walls with irregular thickening. All four exposed rabbits had evidence of pulmonary damage in the form of leukocytic infiltration of the alveolar walls, with or without edema or thickening, and their lungs were unlike those of the control rabbits. Bronchopneumonia was the major cause of delayed death in these animal subjects, especially among the rabbits.

While both Stokinger's and Machle's studies involved much longer durations of exposure than that experienced by the patient, concentrations that were shown to be injurious to animals were below those described by Machle as "tolerable and respirable" and at which "chronic human exposure could easily occur" [Machle and Kitzmiller, 1934].

\section{CONCLUSION}

The patient described in this report suffered hemorrhagic alveolitis and ARDS following use of a household cleaning product containing $8 \%$ hydrofluoric acid. The timing, circumstances of exposure, and clinical features of her illness would suggest HF as the cause of her illness. In our opinion, the presence of HF in a consumer product may be unduly hazardous.

\section{ACKNOWLEDGMENTS}

We acknowledge the helpful comments of Dr. Michael A. MacKinnon. The opinions and conclusions in this paper are those of the authors and are not intended to represent the official position of the Department of Defense, the United States Air Force or any other government agency.

\section{REFERENCES}

ACGIH (1994): “1994-1995 Threshold Limit Values for Chemical Substances and Physical Agents and Biological Exposure Indices." Cincinnati, $\mathrm{OH}$ : American Conference of Governmental Industrial Hygienists.

Alexeeff GV, Lewis DC, Ragle NL (1993): Estimation of potential health effects from acute exposure to hydrogen fluoride using a "benchmark dose" approach. Risk Anal 13:63-69.

Braun J, Stob H, Zober A (1984): Intoxication following the inhalation of hydrogen fluoride. Arch Toxicol 56:50-54.

CaravatiEM(1988):Acutehydrofluoric acidexposure.AmJEmerg Med6:143-150.
Chela A, Reig R, Sanz P, Huguet E, Corbella J (1989): Death due to hydrofluoric acid. Am J Forensic Med Pathol 10:47-48.

Collings GH, Fleming RBL, May R (1951): Absorption and excretion of inhaled fluorides. Ind Hyg Occup Med 4:585-590.

Dieffenbacher PF, Thompson JH (1962): Burns from exposure to anhydrous hydrofluoric acid. J Occup Med 4:325-326.

Dungworth DL (1989): Noncarcinogenic responses of the respiratory track to inhaled toxicants. In McClellan RO, Henderson RF (eds): "Concepts in Inhalation Toxicology." New York: Hemisphere Publishing Corporation. 10, pp. 273-298.

Greendyke RM, Hodge HC (1964): Accidental death due to hydrofluoric acid. J Forensic Sci 9:383-390.

"Handbook of Chemistry and Physics, 59th Ed, 1978-1979." Boca Raton, FL: CRC Press.

Largent EJ (1961): "Fluorosis: The Health Aspects of Fluorine Compounds." Columbus, OH: Ohio State University Press.

Machle W, Kitzmiller K (1934): The effects of the inhalation of hydrogen fluoride. II. The response following exposure to low concentration. J Ind Hyg 17:223-229.

Machle W, Thamann F, Kitzmiller K, Cholak J (1934): The effects of the inhalation of hydrogen fluoride. I. The response following exposure to high concentrations. J Ind Hyg 16:129-145.

MacKinnon MA (1988): Hydrofluoric acid burns. Dermatol Clin 6:67-74.

Mayer L, Guelich J (1963): Hydrogen fluoride (HF) inhalation and burns. Arch Environ Health 7:445-447.

Mayer TG, Gross PL (1985): Fatal systemic fluorosis due to hydrofluoric acid burns. Ann Emerg Med 14:149-153.

Miller FJ, Overton JH, Graham RC (1989): Regional deposition of inhaled reactive gases. In McClellan RO, Henderson RF (eds): "Concepts in Inhalation Toxicology." New York: Hemisphere Publishing Corporation. 8, pp. 229-247.

Morris JB, Smith FA (1982): Regional deposition and absorption of inhaled hydrogen fluoride in the rat. Toxicol Appl Pharmacol 62:81-89.

Reisz GR, Gammon RS (1986): Toxic pneumonitis from mixing household cleaners. Chest 89:49-52.

Sadove R, Hainsworth D, Van Meter W (1990): Total body immersion in hydrofluoric acid. South Med J 83:698-700.

Stavert DM, Archuleta DC, Behr MJ, Lehnert BE (1991): Relative acute toxicities of hydrogen fluoride, hydrogen chloride, and hydrogen bromide in nose- and pseudo-mouth-breathing rats. Fund Appl Toxicol 16:636-655.

Stokinger HE (1949): In Voegtlin C, Hodge HC (eds): "Pharmacology and Toxicology of Uranium Compounds." New York: McGraw-Hill, pp 10211057.

Stokinger HE (1981): The halogens and the nonmetals boron and silica. In Clayton GD, Clayton FE (eds): "Patty's Industrial Hygiene and Toxicology." 3rd revised edition. New York: John Wiley \& Sons. 40, pp. 2937-3043.

Trevino MA, Herrmann GH, Sprout WL (1983): Treatment of severe hydrofluoric acid exposures. J Occup Med 25:861-863.

Waldbott GL, Lee JR (1978): Toxicity from repeated low-grade exposure to hydrogen fluoride-Case report. Clin Toxicol 13:391-402.

Watson A, Oliver JS, Thorpe JW (1973): Accidental death due to inhalation of hydrofluoric acid. Med Sci Law 13:277-279.

White CS, Templeton PA (1992): Chemical pneumonitis. Radiol Clin North Am 30:1231-1243. 